\title{
Preliminary results of rigid fixation (locking plate/screw) after triple pelvic osteotomy without a hip spica cast: A modified fixation method in 21 patients
}

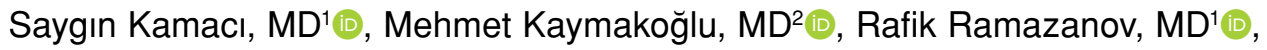 \\ Mehmet Cemalettin Aksoy, MD¹D, Güney Yılmaz, MD'10 \\ ${ }^{1}$ Department of Orthopedics and Traumatology, Hacettepe University Faculty of Medicine, Ankara, Turkey \\ ${ }^{2}$ Department of Orthopedics and Traumatology, Bornova Türkan Özilhan State Hospital, Izmir, Turkey
}

Reorientation of the acetabulum to correct acetabular dysplasia or femoral head subluxation can be achieved by periacetabular, spherical or triple pelvic osteotomies (TPOs) in older ages. Since the periacetabular and spherical osteotomies violates the triradiate cartilage (TRC), they are not recommended in patients with open TRC..$^{[1-5]}$ Conversely, TPO preserves the integrity of the TRC. ${ }^{[6]}$

The TPO was first described by Le Coeur ${ }^{[1]}$ in 1965. Since then, different types of TPO were described and used in developmental dysplasia of the hip (DDH), Legg-Calvé-Perthes disease (LCPD), and neuromuscular hip problems. .7-13] $^{[1}$

Since the posterior column of the pelvis stays intact, periacetabular/spheric osteotomies around

Received: December 04, 2020

Accepted: March 23, 2021

Published online: June 11, 2021

Correspondence: Güney Yılmaz, MD. Hacettepe Üniversitesi Erişkin Hastanesi, Ortopedi ve Travmatoloji Sekreterliği,

06100 Sınhiye, Ankara, Türkiye.

E-mail: aflguney@gmail.com

Doi: 10.52312/jdrs.2021.1

Citation: Kamacı S, Kaymakoğlu M, Ramazanov R, Aksoy MC, Yilmaz $G$. Preliminary results of rigid fixation (locking plate/screw) after triple pelvic osteotomy without a hip spica cast: A modified fixation method in 21 patients. Jt Dis Relat Surg 2021;32(2):454-460.

(O2021 All right reserved by the Turkish Joint Diseases Foundation

This is an open access article under the terms of the Creative Commons Attribution-NonCommercial License, which permits use, distribution and reproduction in any medium, provided the original work is properly cited and is not used for commercial purposes (http://creativecommons.org/licenses/by-nc/4.0/).

\section{ABSTRACT}

Objectives: The aim of this study is to investigate whether rigid fixation after triple pelvic osteotomy (TPO) utilizing a $3.5-\mathrm{mm}$ locking plate and screws without hip spica cast can provide enough stability and prevent correction loss in pediatric patients with developmental dysplasia of the hip (DDH) and Legg-Calvé-Perthes disease (LCPD).

Patients and methods: A total of 21 hips of 21 pediatric patients ( 9 males, 12 females; mean age: $9.3 \pm 2.0$ years; range, 6 to 14 years) who underwent rigid fixation with locking plate/screws after TPO for DDH and LCPD between June 2015 and October 2018 were retrospectively analyzed. Preoperative, immediate postoperative, and six-month follow-up anteroposterior radiographs were compared for the Wiberg's center-edge angle (CE), Sharp angle, acetabular coverage of the femoral head (ACFH), and center-head distance discrepancy (CHDD). The patient demographics, surgery time, perioperative complications were evaluated.

Results: Underlying diagnosis were DDH in 14 patients and LCPD in seven patients. In patients with DDH, postoperative evaluation showed significant increase in the mean CE angle $\left(5.6 \pm 16.1^{\circ}\right.$ vs. $30.5 \pm 9.3^{\circ}$, respectively) and ACFH $(46.4 \pm 16.8 \%$ vs. $84.5 \pm 12.1 \%$, respectively), and a significant decrease in the mean Sharp angle $\left(55.3 \pm 6.2^{\circ} v s .35 .6 \pm 7.8^{\circ}\right.$, respectively) and CHDD $(14.6 \pm 10.7 \%$ vs. $6.2 \pm 5.6 \%$, respectively). The final follow-up revealed that there was no correction loss in these parameters. In the patients with LCPD, postoperative evaluation showed a significant increase in the mean CE $\left(20.1 \pm 11.1^{\circ}\right.$ vs. $38.3 \pm 9.6^{\circ}$, respectively) and ACFH $(62.9 \pm 18 \%$ vs. $91.4 \pm 10.1 \%$, respectively), and a significant decrease in the mean Sharp angle $\left(46 \pm 3.6^{\circ}\right.$ vs. $25.2 \pm 5.5^{\circ}$, respectively). The final follow-up revealed that there was no correction loss in radiological parameters. No perioperative complications were noted.

Conclusion: Our study results suggest that rigid fixation construct with a $3.5-\mathrm{mm}$ locking plate and screws without hip spica cast can provide adequate stability to allow early mobilization following TPO in children without any loss of correction, until bony healing at the osteotomy sites.

Keywords: Early rehabilitation, plate fixation, rigid fixation, triple pelvic osteotomy. 
the acetabulum are relatively stable. Thus, simple fixations methods including pins/screws provides sufficient stability in most of the patients making postoperative immobilization in a cast unnecessary. On the other hand, complete osteotomies around the acetabulum in TPO leaves the acetabulum in an unstable condition and regular fixation methods including screws/pins may need to be supported by a hip spica cast. Although there are also studies without plastering after screw fixation in the literature, no detailed information about the postoperative early rehabilitation, ambulation, and correction loss is available in these series. ${ }^{[6,9,14]}$

Considering that the patients in whom TPO is indicated are in adolescent period or very close to it, postoperative spica cast application makes patient care difficult both for parents and patients. Weight bearing is usually delayed for a minimum of four to six weeks, and no physical therapy can be initiated during this time period. ${ }^{[9,15,16]}$ Additionally, prolonged immobilization increases the risk for deep vein thrombosis and osteopenia and adversely affects muscle strength and hip range of motion (ROM). ${ }^{[17]}$ Non-union of the osteotomy site has been also reported in the literature as a rare complication. ${ }^{[18]}$

In this study, we hypothesized that application of a more rigid fixation with locking plates and screws after TPO would provide sufficient stability in the osteotomy site for early rehabilitation, prevent correction loss, and obviate postoperative hip spica cast application. We, therefore, aimed to investigate whether this type of alternative fixation technique could achieve enough stability and prevent correction loss after TPO, as no other studies focused on these factors.

\section{PATIENTS AND METHODS}

This retrospective case series was conducted at Hacettepe University Faculty of Medicine, Department of Orthopedics and Traumatology between June 2015 and October 2018. A total of 25 patients who underwent TPO with rigid fixation were screened. After exclusion of four patients due to the lost to follow-up and improper radiographs, a total of 21 hips of 21 pediatric patients (9 males, 12 females; mean age: $9.3 \pm 2.0$ years; range, 6 to 14 years) who had TPO with the diagnosis of DDH or LCPD were retrospectively evaluated. All procedures were performed by a fellowship-trained pediatric orthopedic surgeon. Only patients who had standard anteroposterior (AP) pelvic radiographs with minimum six months of follow-up after surgery were included in the study. Those with inadequate follow-up and lack of control radiograph were excluded. The radiological measurements were blindly done twice by a fellowship-trained orthopedic surgeon who was not involved in treatment of the patients. An average of two measurements was accepted for numeric data. A written informed consent was obtained from each parent and/or legal guardians of the patient. The study protocol was approved by the Hacettepe University Faculty of Medicine Ethics Committee (date/no: Go 17/189-38). The study was conducted in accordance with the principles of the Declaration of Helsinki.

Patients' charts were reviewed for demographic data, surgery time and perioperative complications. Preoperative, immediate postoperative, and six-month follow-up radiographs were analyzed for the Wiberg's center-edge angle (CE) ${ }^{[19]}$ Sharp angle, ${ }^{[20]}$ acetabular coverage of the femoral head (ACFH) to assess the coverage of acetabulum over femoral head, and centerhead distance discrepancy (CHDD) ${ }^{[2]}$ to determine the lateralization of the femoral head (Figure 1). The preoperative hips with LCPD were classified according to the Waldenstrom, Catterall, and Lateral Pillar classification systems. ${ }^{[3]}$ Indications for TPO surgery in DDH patients were as follows: eight to 15 years of age, open triradiate cartilage, and an CE angle of $\angle 20^{\circ}$. In LCPD patients, the indications were age above eight years, and Lateral Pillar B or B/C without hinge abduction. The LCPD patients who underwent femoral osteotomy additional to TPO were excluded from the study.

The TPO was performed as previously described by Carlioz et al. ${ }^{[8]}$ through three separate incisions in all patients. Proximal extension of the

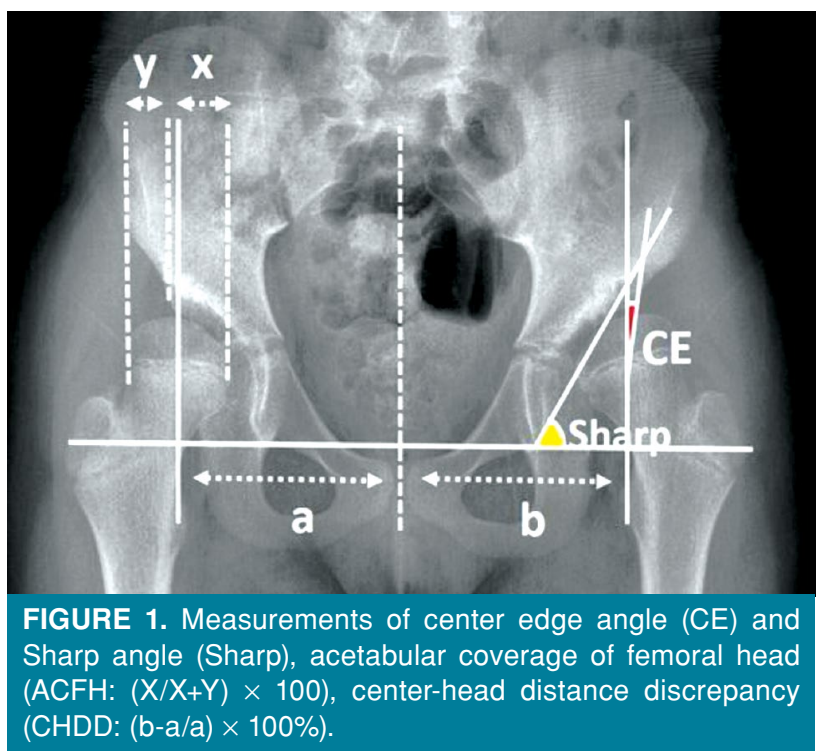




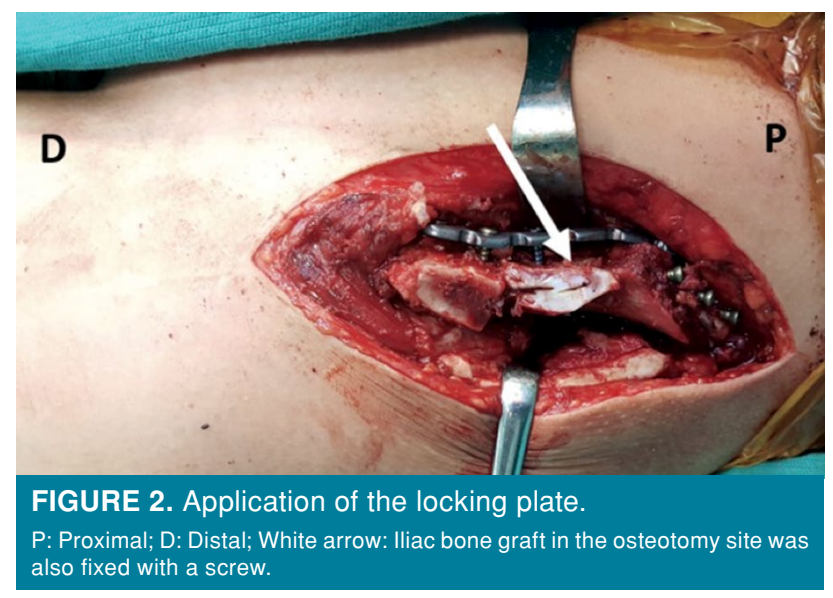

Smith-Peterson approach was utilized to make a Salter type transiliac osteotomy with a Gigli saw. Pubic ramus osteotomy was performed from a vertical skin incision just medial to femoral artery and the nerve. A blunt dissection was performed until the pubic ramus. The surrounding periosteum was cut with a scalpel and an osteotomy with an oscillating saw was performed. Once all the osteotomies were performed, acetabular fragment was rotated anteriorly and laterally to achieve the desired amount of femoral head coverage. The correction was controlled under fluoroscopy and maintained with provisional Kirschner wire (K-wire) fixation. The next step included bending of the stainless-steel reconstruction plate to accommodate over the osteotomy site on the inner surface of the iliac wing. A titanium plate was used, if a stainless-steel plate was not available. A minimum of two locking $3.5 \mathrm{~mm}$ screws were applied in the supraacetabular region and in the proximal iliac wing (Figure 2). Provisional K-wires were, then, removed. Additional fixation with one or two stainless steel cortical screw was applied from proximal fragment to supraacetabular region (Figure 3). The layers were closed in usual manner over a drain. Postoperative spica cast was not applied. The patients were allowed to start bed to chair transfer and gentle hip ROM exercises on postoperative Day one. All patients were allowed to start toe touch non-weight bearing mobilization with crutches in the following days. Postoperative radiographs were obtained at two and six weeks and three and six months. Weight bearing was increased gradually upon the radiographic signs of bony union. The implants were removed in a routine basis 12 months after surgery.
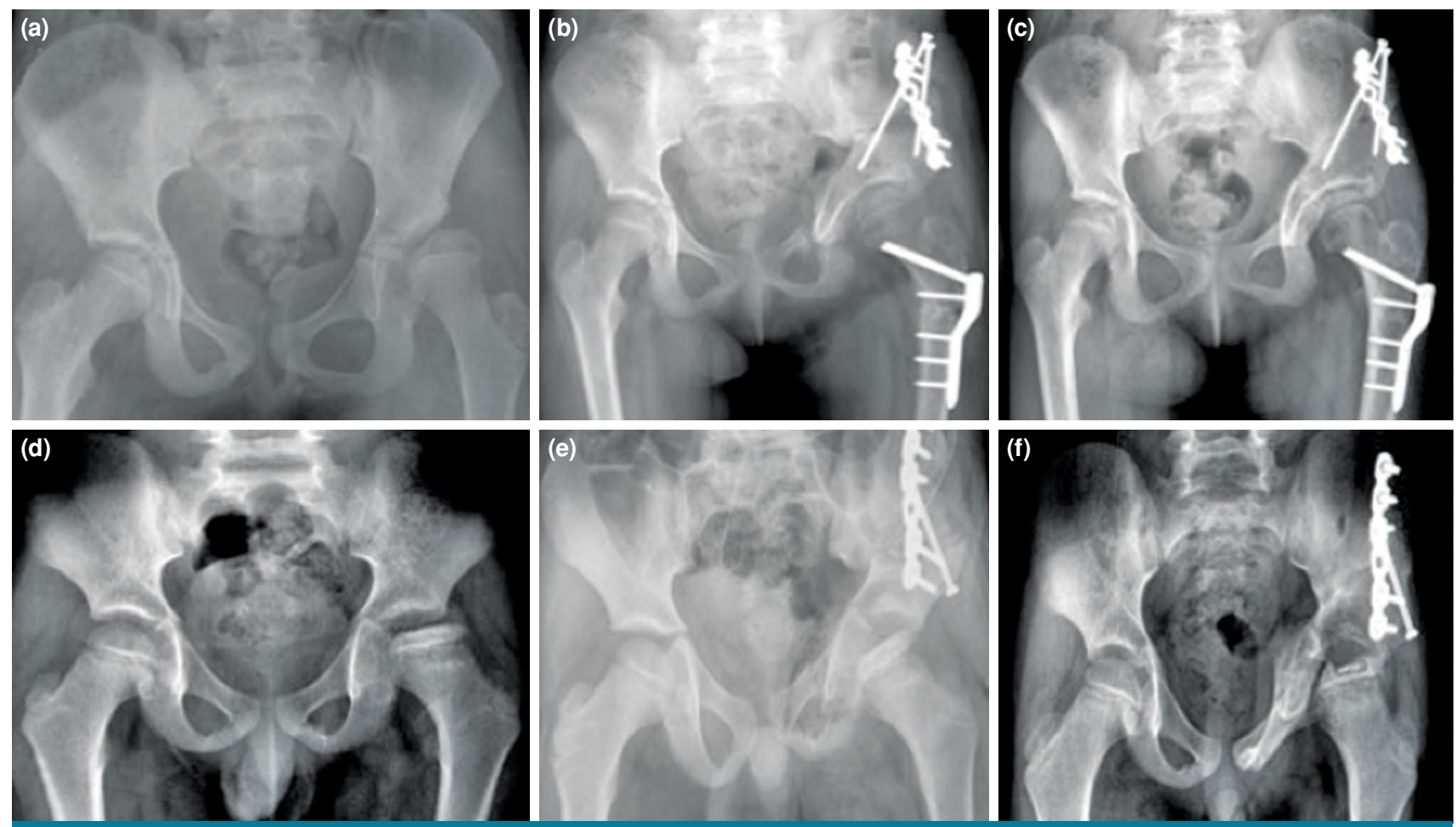

FIGURE 3. (a-c) Preoperative, postoperative, and six-month follow-up radiographs of a nine-year-old female patient with acetabular dysplasia and coxa valga. She underwent proximal femoral osteotomy in addition to TPO. (d-f) Preoperative, postoperative, and six-month follow-up radiographs of a seven-year-old male patient with Legg-Calvé-Perthes disease. 


\begin{tabular}{|c|c|c|c|c|c|}
\hline \multicolumn{6}{|c|}{$\begin{array}{c}\text { TABLE I } \\
\text { ative, and six-month follow-up } r\end{array}$} \\
\hline & Preoperative & Postoperative & Follow-up & $\begin{array}{l}\text { Preoperative vs. } \\
\text { Postoperative }\end{array}$ & $\begin{array}{l}\text { Postoperative vs. } \\
\text { follow-up }\end{array}$ \\
\hline & Mean \pm SD & Mean \pm SD & Mean $\pm S D$ & $p$ & $p$ \\
\hline \multicolumn{6}{|l|}{$\mathrm{DDH}(n=14)$} \\
\hline Center-edge angle $\left({ }^{\circ}\right)$ & $5.6 \pm 16.1$ & $30.5 \pm 9.3$ & $34.9 \pm 10.7$ & $<0.001$ & 0.04 \\
\hline Sharp angle $\left(^{\circ}\right)$ & $55.3 \pm 6.2$ & $35.6 \pm 7.8$ & $36.2 \pm 7.6$ & $<0.001$ & 0.52 \\
\hline ACFH (\%) & $46.4 \pm 16.8$ & $84.5 \pm 12.1$ & $85.9 \pm 12.2$ & $<0.001$ & 0.04 \\
\hline CHDD (\%) & $14.6 \pm 10.7$ & $6.2 \pm 5.6$ & $5.2 \pm 6.2$ & 0.002 & 0.23 \\
\hline \multicolumn{6}{|l|}{ LCPD (n=7) } \\
\hline Center-edge angle $\left(^{\circ}\right)$ & $20.1 \pm 11.1$ & $38.3 \pm 9.6$ & $43.1 \pm 11.2$ & 0.02 & 0.05 \\
\hline Sharp angle $\left(^{\circ}\right)$ & $46 \pm 3.6$ & $25.2 \pm 5.5$ & $27 \pm 5.2$ & 0.02 & 0.6 \\
\hline ACFH (\%) & $62.9 \pm 18$ & $91.4 \pm 10.1$ & $90.6 \pm 16.5$ & 0.02 & 0.89 \\
\hline CHDD (\%) & $2.7 \pm 5.8$ & $2 \pm 7.1$ & $-0.9 \pm 7.3$ & 0.73 & 0.17 \\
\hline
\end{tabular}

\section{Statistical analysis}

Statistical analysis was performed using the IBM SPSS for Windows version 23.0 software (IBM Corp., Armonk, NY, USA). Descriptive data were expressed in mean \pm standard deviation (SD), median (min-max) or number and frequency, where applicable. The normality analysis of the data was performed using the Kolmogorov-Smirnov test. The mean values of the normally distributed values were evaluated using the paired t-test and the mean of the variables that did not show normal distribution was evaluated using the Wilcoxon test. A $p$ value of 0.05 was considered statistically significant.

\section{RESULTS}

Underlying diagnosis were DDH in 14 patients (3 boys, 11 girls) and LCPD in seven patients (6 boys, 1 girl). The mean follow-up was $24 \pm 10.5$ (range, 6 to 38) months. The left hip was operated in 13 patients and the right side in eight patients. The mean surgery time was $163.5 \pm 33$ (range, 120 to 225) min. A simultaneous ipsilateral proximal femoral varus derotation osteotomy was performed in six patients with DDH and ipsilateral adductor tenotomy was performed for abduction deficit in two patients with LCPD.

In 14 patients with $\mathrm{DDH}$, the mean $\mathrm{CE}$ angle was $5.6 \pm 16.1^{\circ}$ preoperatively and $30.5 \pm 9.3^{\circ}$ postoperatively. The increase in the $\mathrm{CE}$ angle was statistically significant after surgery $(\mathrm{p}<0.001)$. The mean CE angle at six months of follow-up was $34.9 \pm 10.7^{\circ}$. The mean CE angle increased significantly at six months, compared to postoperative value $(\mathrm{p}=0.04)$. The mean preoperative Sharp angle decreased from $55.3 \pm 6.2^{\circ}$ to $35.6 \pm 7.8^{\circ}$ postoperatively $(\mathrm{p}<0.001)$. The mean Sharp angle at six months was $36.2 \pm 7.6^{\circ}(\mathrm{p}=0.52)$. The mean ACFH significantly increased from $46.4 \pm 16.8 \%$ to $84.5 \pm 12.1 \%$ postoperatively ( $\mathrm{p}<0.001)$. At six months of follow-up, the mean ACFH was $85.9 \pm 12.2 \%(p=0.04)$. The mean CHDD was $14.6 \pm 10.7 \%$ preoperatively, $6.2 \pm 5.6 \%$ postoperatively, and $5.2 \pm 6.2 \%$ at six months. There was a statistically significant decrease in the CHDD on postoperative radiographs compared to baseline $(p=0.002)$. However, the mean CHDD at six months was not significantly different showing that the operative correction was preserved at six months in DDH patients (Table I).

In seven patients with LCPD, the mean CE angle was $20.1 \pm 11.1^{\circ}$ preoperatively and $38.3 \pm 9.6^{\circ}$ postoperatively $(p=0.02)$. The mean $C E$ angle at six months was $43.1 \pm 11.2^{\circ}(\mathrm{p}=0.05)$. The mean Sharp angle decreased from $46 \pm 3.6^{\circ}$ to $25.2 \pm 5.5^{\circ}$ postoperatively $(\mathrm{p}=0.02)$. The mean Sharp angle at six months was $27 \pm 5.2^{\circ}$, which was similar to the postoperative value $(\mathrm{p}=0.6)$. The mean ACFH increased from $62.9 \pm 18 \%$ to $91.4 \pm 10.1 \%$ postoperatively ( $\mathrm{p}=0.02$ ). The mean CHDD was measured as $2.7 \pm 5.8$ on preoperative, $2 \pm 7.1 \%$ on postoperative, and $-0.9 \pm 7.3 \%$ on six-month follow-up radiographs. The mean CHDD on preoperative, postoperative, and six-month follow-up radiographs was not significantly different, showing that the operative correction was preserved at six months in LCPD patients (Table I).

Of 14 patients with $\mathrm{DDH}$, four suffered from bilateral disease. None of the patients had previous 
pelvic osteotomies. Of seven patients with LCPD, one had bilateral disease. According to the Waldenstrom classification, three patients were in the fragmentation stage, two patients were in the reossification stage, and two patients in the remodeling stage. According to the Lateral Pillar classification three patients were considered as Group B and four patients were considered as Group C. Although we had five patients with bilateral hip pathology, none of them underwent bilateral hip surgery. Contralateral side of the patient with LCPD were managed conservatively, and all of our DDH patients were on schedule to have a TPO for contralateral side in the next year. Our short follow-up period was limited to include the second procedure and its outcomes.

No complications including infection, neurovascular injury, joint stiffness, skin necrosis, ileus or loosening of the fixation were observed in the perioperative period. Radiographic union of the osteotomy sites was observed in all patients at six to eight weeks of follow-up.

\section{DISCUSSION}

Our findings suggested that rigid fixation construct with a 3.5-mm locking plate/screws without hip spica cast provided adequate stability to allow early mobilization following TPO in children without any loss of correction, until bony healing at the osteotomy sites.

In recent years, there is an increasing trend toward containment surgeries in DDH and LCPD, when conservative treatment options fail. The main goals of TPO in DDH are to improve the congruence of the hip joint and to correct acetabular dysplasia. Thus, symmetric load distribution is achieved, stability of the hip joint is increased, and mechanical environment is improved. The TPO aims to correct femoral head subluxation in LCPD, which is crucial to obtain a round femoral head in the long-term. Detrimental effects of LCPD and DDH on hip joint can be prevented, as long as these goals are achieved. ${ }^{[2-4,21]}$

The TPO enables to improve anterolateral coverage of the femoral head. The effectiveness of the procedure has been shown in various studies with different radiological parameters such as CE angle, Sharp angle, and ACFH. . $^{[4,6,9,11,14,17,20]}$ We found $22.7^{\circ}$ increase in the mean $\mathrm{CE}$ angle, 34.9\% increase in the mean $\mathrm{ACFH}$, and $20^{\circ}$ decrease in the mean Sharp angle following TPO, which is compatible with the existing literature. Although these increases were mainly due to correction maneuvers intraoperatively, we also observed a further slight improvement in the mean CE angle at six months of follow-up, compared to immediate postoperative values. This was surprising at the first sight due to the rigid fixation method we used; however, a slightly improvement in the CE angle after pelvic osteotomies until the skeletal maturity were reported in some other studies. ${ }^{[5,22]}$ This further improvement in the CE angle may be triggered by remodeling capacity until skeletal maturity.

The TPO is a relatively unstable osteotomy, since the posterior and anterior columns are violated through complete osteotomies. Fixation of the reoriented acetabulum is obtained most commonly with K-wires or screws. ${ }^{[--8,14,15,17]}$ However, that type of fixation does not provide enough stability for early rehabilitation and postoperative hip spica casting is required in most of the patients for six to eight weeks. There is no consensus in the literature on the use of hip spica. Prolonged immobilization in a cast deteriorates patient comfort, perineal hygiene and it is inconvenient for caregivers. Additionally, the risk of cast-related complications including skin necrosis and ileus are increased. ${ }^{[6,14]}$ Moreover, casting does not eliminate all the forces acting on the hip and daily activities of the child in the cast may cause displacement at the osteotomy site. Various fixation strategies were described following TPO: ${ }^{[10,23]}$ Steel ${ }^{[6]}$ described his operative technique in a series of patients aged between seven to 17 years. Fixation was achieved via two extra-articular pins penetrating to the inner tabula of iliac wing. Additional hip spica cast was maintained for a period of eight to 10 weeks. Passive and active ROM exercises were started following cast removal and weight bearing was allowed with crutches at 12 to 14 weeks. In their cohort aged between 9 to 32 years, Tönnis et al. ${ }^{[9]}$ achieved fixation with a bone wedge taken from the iliac crest 4 to 6-cm proximal to anterior superior iliac spine. The graft was placed into the osteotomy site which was supported by four non-threaded 2-2.5 mm K-wires. The patients were immobilized in a hip spica cast for six weeks. Weight bearing was delayed, until the consolidation of the osteotomy sites which occurred at 12 to 16 weeks. Vukasinovic et al. ${ }^{[15]}$ published 76 hips at a mean age of 15.9 years following TPO. Fixation was achieved via two or three K-wires. Postoperative skin traction was applied to all cases for a mean of $44.4 \pm 8.2$ days. Weight bearing allowed after a mean of $128.7 \pm 32.3$ days. There are also studies that applied more rigid fixation than K-wires, such as screws. Farsetti et al. ${ }^{[24]}$ applied Tonnis type TPO in 54 patients with an older mean age (16.8 years), allowed earlier 
mobilization, and reported good clinical results with five asymptomatic ischial non-unions. A more rigid construct with plate would have been useful to avoid non-unions.

Postoperative comfort and early rehabilitation have become important parameters for patient satisfaction and success after surgery. Fixation techniques which obviate the use prolonged immobilization or casting have increasingly gained popularity in pediatric orthopedic surgery to achieve these goals. On the other hand, radiological correction and maintaining this correction throughout the postoperative period is a necessity, while improving patient comfort. None of our patients experienced clinical problems such as infection, wound problems and loss of fixation related to the plate fixation. We believe that our new approach in TPO fixation utilizing locking plates can provide sufficient stability for early rehabilitation without correction loss. This increased stability may also avoid possible non-union of osteotomy sites in TPO previously reported in several studies. ${ }^{[25,26]}$ Ischial and pubic osteotomy site non-unions are hard to manage and we had no non-unions in our study group, neither at iliac bone nor ischium/pubic bone, also with the possible contribution of the young mean age.

There are some limitations to this study. It has a retrospective design without a control group. The number of patients is also small, and the study included two groups of patients with different diagnoses. Additionally, the follow-up period was relatively short. Since the aim of the study was to present our surgical technique and investigate whether rigid fixation in TPO prevented correction loss in the short-term, we believe that the number of patients and follow-up period is adequate. Besides, bony union occurred in the osteotomy sites of all patients in our study during six-month follow-up, indicating that it is very unlikely to experience loss of correction in the future. However, a longer followup would have been useful to assess the clinical outcomes of our technique. We routinely remove the hardware one year after surgery.

In conclusion, our findings suggest that rigid fixation construct with a 3.5-mm locking plate/ screws without hip spica cast provides adequate stability to allow early mobilization following TPO in children without any loss of correction, until bony healing at the osteotomy sites. However, further large-scale, long-term, prospective, clinical studies are needed to evaluate the clinical relevance of this technique.

\section{Declaration of conflicting interests}

The authors declared no conflicts of interest with respect to the authorship and/or publication of this article.

\section{Funding}

The authors received no financial support for the research and/or authorship of this article.

\section{REFERENCES}

1. Le Coeur P. Orientation of the hip joint by osteotomy of the ischium and ilium. Rev Chir Orthop 1965;51:211-212.

2. Chen IH, Kuo KN, Lubicky JP. Prognosticating factors in acetabular development following reduction of developmental dysplasia of the hip. J Pediatr Orthop 1994;14:3-8.

3. Herring JA, Kim HT, Browne R. Legg-Calve-Perthes disease. Part II: Prospective multicenter study of the effect of treatment on outcome. J Bone Joint Surg [Am] 2004;86:2121-34.

4. Peters CL, Fukushima BW, Park TK, Coleman SS, Dunn HK. Triple innominate osteotomy in young adults for the treatment of acetabular dysplasia: A 9-year follow-up study. Orthopedics 2001;24:565-9.

5. Terjesen T, Horn J. Management of late-detected DDH in children under three years of age: 49 children with followup to skeletal maturity. Bone Jt Open 2020;1:55-63.

6. Steel HH. Triple osteotomy of the innominate bone. 1973. J Bone Joint Surg [Am] 2004;86:644.

7. de Kleuver M, Kooijman MA, Pavlov PW, Veth RP. Triple osteotomy of the pelvis for acetabular dysplasia: Results at 8 to 15 years. J Bone Joint Surg Br 1997;79:225-9.

8. Carlioz H, Khouri N, Hulin P. Ostéotomie pelvienne triple juxta-cotyloïdienne. Rev Chir Orthop 1982;68:497-501.

9. Tönnis D, Behrens K, Tscharani F. A modified technique of the triple pelvic osteotomy: Early results. J Pediatr Orthop 1981;1:241-9.

10. Eren A, Omeroglu H, Güven M, Ugutmen E, Altintas F. Incomplete triple pelvic osteotomy for the surgical treatment of dysplasia of the hip in adolescents and adults. J Bone Joint Surg [Br] 2005;87:790-5.

11. Camurcu IY, Yildirim T, Buyuk AF, Gursu SS, Bursali A, Sahin V. Tönnis triple pelvic osteotomy for Legg-CalvePerthes disease. Int Orthop 2015;39:485-90.

12. Vukasinovic Z, Spasovski D, Vucetic C, Cobeljic G, Zivkovic Z, Matanovic D. Triple pelvic osteotomy in the treatment of Legg-Calve-Perthes disease. Int Orthop 2009;33:1377-83.

13. Conroy E, Sheehan E, O' Connor P, Connolly P, McCormack D. Triple pelvic osteotomy in Legg-Calve-Perthes disease using a single anterolateral incision: A 4-year review. J Pediatr Orthop B 2010;19:323-6.

14. Janssen D, Kalchschmidt K, Katthagen BD. Triple pelvic osteotomy as treatment for osteoarthritis secondary to developmental dysplasia of the hip. Int Orthop 2009;33:1555-9.

15. Vukasinovic Z, Pelillo F, Spasovski D, Seslija I, Zivkovic Z, Matanovic D. Triple pelvic osteotomy for the treatment of residual hip dysplasia. Analysis of complications. Hip Int 2009;19:315-22.

16. Lipton GE, Bowen JR. A new modified technique of triple osteotomy of the innominate bone for acetabular dysplasia. Clin Orthop Relat Res 2005;(434):78-85. 
17. van Hellemondt GG, Sonneveld $H$, Schreuder $M H$, Kooijman MA, de Kleuver M. Triple osteotomy of the pelvis for acetabular dysplasia: Results at a mean follow-up of 15 years. J Bone Joint Surg [Br] 2005;87:911-5.

18. Özcan Ö, Yeşil M, Altın R. Triple nonunion after steellike triple pelvic osteotomy. Eklem Hastalik Cerrahisi 2018;29:193-7.

19. Wiberg G. Shelf operation in congenital dysplasia of the acetabulum and in subluxation and dislocation of the hip. J Bone Joint Surg [Am] 1953;35-A:65-80.

20. Sharp IK. Acetabular Dysplasia-the Acetabular Angle. J Bone Joint Surg [Br] 1961;43:268-72.

21. Wenger DR, Pring ME, Hosalkar HS, Caltoum CB, Lalonde FD, Bastrom TP. Advanced containment methods for LeggCalvé-Perthes disease: results of triple pelvic osteotomy. J Pediatr Orthop 2010;30:749-57.

22. EzirmikN, Yildız K. Salter innominate osteotomy or Pemberton pericapsular osteotomy in treatment of developmental dysplasia of hip. Turk J Med Sci 2012;42:1058-62.
23. Rahimi H, Kachooei AR, Hallaj-Moghaddam M, Gharedaghi M, Mirkazemi M, Shahpari O, et al. A modified triple pelvic osteotomy for the treatment of hip hypoplasia. Arch Bone Jt Surg 2013;1:31-4.

24. Farsetti P, Caterini R, De Maio F, Potenza V, Efremov $\mathrm{K}$, Ippolito E. Tönnis triple pelvic osteotomy for the management of late residual acetabular dysplasia: Midterm to long-term follow-up study of 54 patients. J Pediatr Orthop B 2019;28:202-6.

25. Tschauner C, Sylkin A, Hofmann S, Graf R. Painful nonunion after triple pelvic osteotomy. Report of five cases. J Bone Joint Surg [Br] 2003;85:953-5.

26. Yilmaz E, Damla H, Norvell DC, Kalchschmidt K, Luering C, Zahedi AR. Risk factors associated with non-union after triple pelvic osteotomy (Toennis and Kalchschmidt technique): A case-control study and review of the literature. Arch Orthop Trauma Surg 2019;139:173-80. 